\section{Defining Chronic Pain}

\section{To the Editor:}

We read with interest the article by Taylor, et al, "Is Chronic Pain a Disease in Its Own Right? Discussions from a Pre-OMERACT 2014 Workshop on Chronic Pain," published in The Journal of Rheumatology ${ }^{1}$.

First, we are quite amazed to see how widespread the attendees' responses were regarding the definition of chronic pain $(\mathrm{CP})$ after a few presentations were given, including 1 proposing that $\mathrm{CP}$ should no longer be seen as a symptom. Out of the 5 choices given for the definition of $\mathrm{CP}$, $9 \%$ selected "disease," $26 \%$ selected "condition," $11 \%$ selected "syndrome," $23 \%$ selected "symptom complex," and 31\% selected "none of the above." It is somewhat difficult to accept that only $9 \%$ of attendees chose "disease" during the pre-work survey at the workshop, where "Is chronic pain a disease in its own right?" was supposed to be the main objective of this meeting.

We wonder if this result had to do with the ambiguous definition of the word "disease."

Merriam-Webster Medical Dictionary defines "disease" as "an impairment of the normal state of the living animal or plant body or one of its parts that interrupts or modifies the performance of the vital functions, is typically manifested by distinguishing signs and symptoms, and is a response to environmental factors (as malnutrition, industrial hazards, or climate), to specific infective agents (as worms, bacteria, or viruses), to inherent defects of the organism (as genetic anomalies), or to combinations of these factors"2.

According to the Oxford English Dictionary, cited in the paper by Taylor, et al, "disease" is defined as a disorder of structure or function in a human, animal, or plant, especially one that produces specific symptoms or that affects a specific location and is not simply a direct result of physical injury ${ }^{1}$.

Wikipedia defines "disease" as "a particular abnormal condition, a disorder of a structure or function, that affects part or all of an organism"3.

Regardless of the exact definition of $\mathrm{CP}$, we think the term "longterm condition" should be avoided because it fails to voice the urgent need and justification to treat those patients who experience CP. In a recent article by Paulus, he opined that "[the] opportunity for neuroscience-based approaches to impact clinical psychiatry has never been greater, yet biological measures have had almost no impact on psychiatric practice" ${ }^{\text {. We wonder how much }}$ this statement applies to pain medicine, especially to the clinical practice of $\mathrm{CP}$ management. Thus, defining $\mathrm{CP}$ in a scientific yet practical way can help advance the field of $\mathrm{CP}$ management, and help patients, clinicians, researchers, healthcare policy makers, and patients' friends and family members.

In this regard, we agree with Argoff, et al (not referenced in the article by Taylor, et al) that "Chronic pain is a multifaceted disease [...] Elucidating mechanisms that govern normal sensation in the periphery has provided insights into the biochemical, molecular, and neuroanatomic correlates of chronic pain, an understanding of which is leading increasingly to mechanism-specific multidrug therapies. Peripheral and central neuroplastic reorganization underlying the disease of chronic pain is influenced by patient-specific emotions, cognition, and memories, further impairing function and idiosyncratically defining the illness of chronic pain" 5 .

Further, we believe that while CP is multifaceted, as pointed out by Argoff, et al, it may be regarded as having different stages during its course/progression. We see numerous examples: some patients with $\mathrm{CP}$ can still be completely functional, while others are completely disabled. Our speculation is that the $\mathrm{CP}$ population falls into a spectrum: at one end, representing those with manageable pain levels and totally functional, and at the other end representing those with intractable pain with various manifesting comorbid conditions and completely disabled. We would consider somewhere along the spectrum toward the end as completely disabled, the $\mathrm{CP}$ definition changing from "disease" to "CP syndrome."

The major advantages of using the "multifaceted disease" model of $\mathrm{CP}$ are (1) the positive attitude that patients with CP can be helped, (2) promotion of treatment compliance from patients and providers' effort in helping patients, (3) the use of a multidisciplinary approach, and (4) having healthcare policy makers and insurance companies provide coverage-related treatment modalities.

We believe, at this point, that $\mathrm{CP}$ as a multifaceted disease model, with potential to deteriorate into full-blown "chronic pain syndrome," is both scientific and pragmatic. Our job as pain care providers is to do anything within our power to help patients with $\mathrm{CP}$ and prevent them from experiencing CP syndrome.

XIULU RUAN, MD, Adjunct Clinical Associate Professor of Anesthesia, Department of Anesthesiology, Louisiana State University Health Science Center; ALAN DAVID KAYE, MD, PhD, Professor and Chairman of Anesthesia, Department of Anesthesiology, Louisiana State University Health Science Center, New Orleans, Louisiana, USA. Address correspondence to Associate Professor X. Ruan, Anesthesiology, Louisiana State University Health Science Center, 1542 Tulane Ave., New Orleans, Louisiana 70112,USA.E-mail: drxruan88@gmail.com

\section{REFERENCES}

1. Taylor AM, Phillips K, Taylor JO, Singh JA, Conaghan PG, Choy $\mathrm{EH}$, et al. Is chronic pain a disease in its own right? Discussions from a pre-OMERACT 2014 workshop on chronic pain. J Rheumatol 2015;42:1947-53.

2. Merriam-Webster Dictionary. Definition of disease. [Internet. Accessed December 18, 2015.] Available from: www.merriam-webster.com/dictionary/disease

3. Wikipedia. Definition of disease. [Internet. Accessed December 30, 2015.] Available from: en.wikipedia.org/wiki/Disease

4. Paulus MD. Pragmatism instead of mechanism: a call for impactful biological psychiatry. JAMA Psychiatry 2015;72:631-2.

5. Argoff CE, Albrecht P, Irving G, Rice F. Multimodal analgesia for chronic pain: rationale and future directions. Pain Med 2009;10 Suppl 2:S53-66.

J Rheumatol 2016;43:4; doi:10.3899/jrheum.150807 